\title{
Disk Heating, Galactoseismology, and the Formation of Stellar Halos
}

\author{
Kathryn V. Johnston ${ }^{1, *, \dagger}$, Adrian M. Price-Whelan ${ }^{2, \dagger}$, Maria Bergemann ${ }^{3}$, Chervin Laporte ${ }^{1}$, \\ Ting S. Li ${ }^{4}$, Allyson A. Sheffield ${ }^{5}$, Steven R. Majewski ${ }^{6}$, Rachael S. Beaton ${ }^{7}$, Branimir Sesar ${ }^{3}$ \\ and Sanjib Sharma ${ }^{8}$ \\ 1 Department of Astronomy, Columbia University, 550 W 120th st., New York, NY 10027, USA; \\ cfl2126@columbia.edu \\ 2 Department of Astrophysical Sciences, Princeton University, 4 Ivy Lane, Princeton, NJ 08544, USA; \\ adrn@astro.princeton.edu \\ 3 Max Planck Institute for Astronomy, Heidelberg 69117, Germany; bergemann@mpia-hd.mpg.de (M.B.); \\ bsesar@mpia.de (B.S.) \\ 4 Fermi National Accelerator Laboratory, P. O. Box 500, Batavia, IL 60510, USA; sazabi@neo.tamu.edu \\ 5 Department of Natural Sciences, LaGuardia Community College, City University of New York, \\ 31-10 Thomson Ave., Long Island City, NY 11101, USA; asheffield@lagcc.cuny.edu \\ 6 Department of Astronomy, University of Virginia, P.O. Box 400325, Charlottesville, VA 22904, USA; \\ srm4n@virginia.edu \\ 7 The Carnegie Observatories, 813 Santa Barbara Street, Pasadena, CA 91101, USA; rlb9n@virginia.edu \\ 8 Sydney Institute for Astronomy, School of Physics, University of Sydney, NSW 2006, Australia; \\ sanjib.sharma@gmail.com \\ * Correspondence: kvj@astro.columbia.edu; Tel.: +1-212-854-3884 \\ + These authors contributed equally to this work.
}

Academic Editors: Duncan A. Forbes and Ericson D. Lopez

Received: 1 July 2017; Accepted: 14 August 2017; Published: 26 August 2017

\begin{abstract}
Deep photometric surveys of the Milky Way have revealed diffuse structures encircling our Galaxy far beyond the "classical" limits of the stellar disk. This paper reviews results from our own and other observational programs, which together suggest that, despite their extreme positions, the stars in these structures were formed in our Galactic disk. Mounting evidence from recent observations and simulations implies kinematic connections between several of these distinct structures. This suggests the existence of collective disk oscillations that can plausibly be traced all the way to asymmetries seen in the stellar velocity distribution around the Sun. There are multiple interesting implications of these findings: they promise new perspectives on the process of disk heating; they provide direct evidence for a stellar halo formation mechanism in addition to the accretion and disruption of satellite galaxies; and, they motivate searches of current and near-future surveys to trace these oscillations across the Galaxy. Such maps could be used as dynamical diagnostics in the emerging field of "Galactoseismology", which promises to model the history of interactions between the Milky Way and its entourage of satellites, as well examine the density of our dark matter halo. As sensitivity to very low surface brightness features around external galaxies increases, many more examples of such disk oscillations will likely be identified. Statistical samples of such features not only encode detailed information about interaction rates and mergers, but also about long sought-after dark matter halo densities and shapes. Models for the Milky Way's own Galactoseismic history will therefore serve as a critical foundation for studying the weak dynamical interactions of galaxies across the universe.
\end{abstract}

Keywords: galaxies: galaxy formation; galactic disks; stellar halos; Milky Way 


\section{Introduction}

Our perspective on the Milky Way presents both unique challenges and unique opportunities within our quest to understand galaxies more generally. Because we are located inside of our own Galaxy, it is the only galaxy for which we lack a truly global perspective in a single snapshot and, instead, must survey the entire night sky to fully sample its constituents. On the other hand, it is one of the few galaxies that we can presently study by individual stars and it is the only galaxy for which we can make volume-complete samples in both position and velocity space for non-evolved stellar tracers (e.g., via Main Sequence Turnoff Stars, hereafter MSTO). Present and recent sky surveys have already considerably advanced this effort, and surveys in the near future will deliver massive datasets that will enable detailed studies of stellar structures throughout the Galactic volume.

Emerging in the 1990s, the catalogues that provided the inspiration for current and future surveys not only mapped global structures in our Galaxy, but also revealed the ubiquity of substructure within it. These revelations added an unforeseen richness to interpretations of the data sets and encouraged the development of new dynamical tools for studying ongoing interactions and formation histories. As a few examples:

- Astrometric data from the Hipparcos mission [1] led to the discovery of moving groups in the velocity distribution of solar-neighborhood stars [2]. Some of these likely correspond to destroyed star clusters (as expected), while others (unexpectedly) have been interpreted as signatures of resonances with the Galactic bar [3].

- $\quad$ Precise, large-area photometry from the Sloan Digital Sky Survey (hereafter, SDSS - [4-6]) led to the discovery of many "streams" of MSTO stars in the Galactic stellar halo. These are understood to be the remnants of long-dead satellite galaxies and dissolved globular clusters [7,8] and serve as a stunning confirmation that our Galaxy has indeed formed hierarchically (e.g., [9-11]).

- All-sky, infrared photometry from the Two Micron All Sky Survey (hereafter, 2MASS - [12]) enabled M giant stars associated with tidal debris from the Sagittarius dwarf galaxy (hereafter Sgr) to be traced around the entire sky [13], offering a new perspective on the history of its disruption [14].

From these and many other studies using large survey catalogues, it is now clear that the Milky Way is full of kinematic substructure, from the nearby regions of the Galactic disk to the distant stellar halo.

The focus of this Article is on three such substructures just beyond the historical "end" of the Galactic disk within the inner stellar halo. Figure 1 (reproduced with data from previous work, [15]), shows the spatial distribution of M giant stars associated with these three substructures: the so-called Monoceros Ring (Mon; also known as the Galactic Anticenter Stellar Structure, GASS), the Triangulum-Andromeda clouds (TriAnd), and A13. 

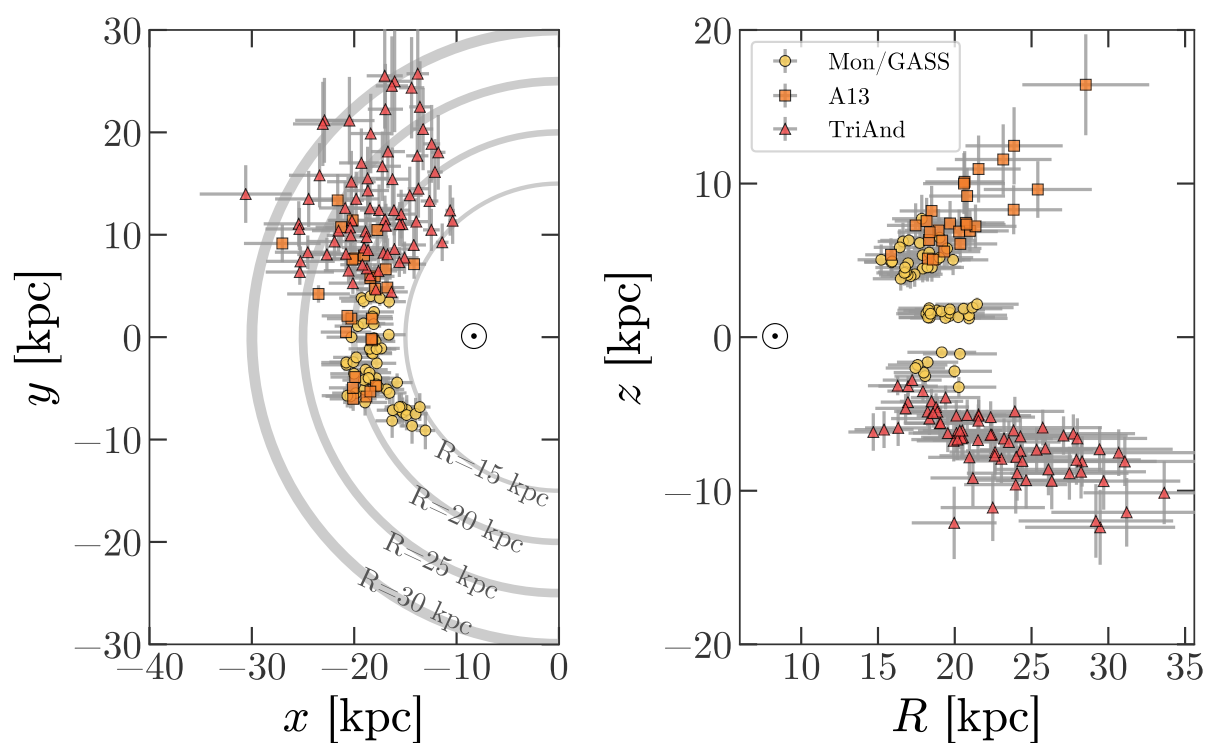

Figure 1. Summary of the spatial distribution of $M$ giants in each of the three low-latitude structures. Note that at lower Galactic latitudes, the lack of candidate $\mathrm{M}$ giant members is due to selection effects and crowding near the midplane; we expect the structures to continue towards even lower latitudes, but blend with ordinary disk stars. Markers represent individual stars identified as likely members of each of the three structures discussed in this work (see figure legend). Distance estimates come from photometry alone and have expected absolute uncertainties around $\approx 20 \%$ for TriAnd and A13 [15,16] and $\approx 25 \%$ for Mon/GASS. Grey curves in left panel show Galactocentric circles with cylindrical radii, $R$, indicated on the figure. The position of the Sun is marked with the solar symbol $\odot$.

Each of these substructures were originally identified as over-densities in stellar number counts relative to the expected global structure of the disk or inner stellar halo ${ }^{1}$. The same region of the sky has been shown to be richly structured on even smaller scales [19-23], but here we consider only the larger structures. Unlike most stellar streams, Mon/GASS, TriAnd, and A13 are present at a range of low to moderate Galactic latitudes and span large areas of the sky-we will hereafter refer to them collectively as the "low-latitude structures". The basic properties of the low-latitude structures are summarized below:

- Mon/GASS is an arc-like or partial-ring feature of stars beyond the previously-expected edge of the Galactic disk, $\approx 5 \mathrm{kpc}$ beyond the Sun in cylindrical radius [24]. Stars attributed to Mon/GASS span a large area of the sky and large range in distance: Galactic longitudes $\approx 120^{\circ} \lesssim l \lesssim 240^{\circ}$, latitudes $-30^{\circ} \lesssim b \lesssim+40^{\circ}$, and heliocentric distances $5 \lesssim d_{\odot} \lesssim 10 \mathrm{kpc}[17,18,25]$. Radial velocity measurements of $\mathrm{M}$ giant stars associated with the structure follow a clear trend in mean velocity with Galactic longitude and have a velocity dispersion much smaller than the stellar halo [26].

- TriAnd was first discovered as a diffuse over-density of $\mathrm{M}$ giant stars covering the area $\approx 100^{\circ} \lesssim l \lesssim 160^{\circ}, \approx-35^{\circ} \lesssim b \lesssim-15^{\circ}$, overlapping the Mon/GASS structure on the sky but at larger heliocentric distances of $\approx 15-25 \mathrm{kpc}$ [27]. The M giants again exhibit a coherent radial-velocity sequence with a dispersion much smaller than the halo [27]. Deep photometry in the region revealed MSTO stars associated with the structure and proposed the existence of a second main sequence ("TriAnd 2") at larger distance, $\approx 30-35 \mathrm{kpc}[22,28]$.

- $\quad A 13$ is another tenuous association of M giants in the North Galactic Hemisphere in the area $\approx 125^{\circ} \lesssim l \lesssim 210^{\circ}, \approx 20^{\circ} \lesssim b \lesssim 40^{\circ}$ at approximate distances of $\approx 10-20 \mathrm{kpc}$. It was initially

1 Here we show just M giant stars that have been previously identified as candidate members of the structures, however, some of the structures have also been detected in MSTO stars $[17,18]$. 
discovered by applying a group finding algorithm [29] to all M giants in the 2MASS photometric catalogue [30]. Again, radial velocities of $\mathrm{M}$ giants in this structure have a small velocity dispersion around a roughly linear trend with Galactic longitude [15]

Several distinct scenarios have been used to explain the formation and existence of each of these structures. Mon/GASS has been attributed to the accretion of a satellite [31], a natural extension of the Galactic warp [32,33], or disturbances to the Galactic disk [21,34-38]. The extreme position of TriAnd at $(R, Z) \approx(15,-5)$ to $(25,-12) \mathrm{kpc}$ across a large range in Galactocentric azimuth, $\phi$, seemed to exclude the possibility of a disturbed Galactic disk as a possible origin and it has also been modeled as debris from a satellite on a retrograde orbit [16]. Initial abundance studies measured $[\alpha / \mathrm{Fe}]$ and s-process values for M giants in both Mon/GASS and TriAnd and found them to be consistent with those seen in Milky Way satellite galaxies, thus unlike the disk [39,40] - see Section 2.3 for a more complete discussion.

Recent evidence points towards a more convincing, coherent picture for the nature of the three low-latitude structures: the stars in these structures likely have a common origin in the Galactic disk and have been "kicked out" to their present-day positions. This Article reviews recent and ongoing contributions that our own group is making to formulating this picture, which include spectroscopic surveys of the low-latitude structures to study metallicities and kinematic properties [15,16], stellar populations (Sheffield et al., in prep [41]), and detailed abundance patterns (Bergemann et al., in prep.), as well as numerical simulations (Laporte et al., in prep [16,41,42]). We summarize this observational and theoretical work in Sections 2 and 3 respectively, adding in the context of contemporary work from other groups, as well as the larger context of possible connections across the Galactic disk. Armed with this understanding of the nature of these substructures, we proceed in Section 4 to discuss prospects for mapping such structures more generally around our own and other galaxies. We end in Section 5 by outlining the motivation for making such maps, asking what they might be telling us about bigger questions: galaxy formation scenarios and the distribution of dark matter around galaxies.

\section{The Nature of Structures Around the Outer Disk-Summary of Observations}

From clustering in positions or distance alone, many candidate groups and over-densities of $\mathrm{M}$ giants have been identified in the outer disk or inner halo. Over the last five years our group has obtained spectroscopy for candidate members of these structures with the aims of (1) confirming the existence of substructure in velocities, (2) measuring chemical abundances, and (3) studying the constituent stellar populations. These goals then inform our own efforts to produce plausible dynamical formation scenarios using simulations. In particular, we avoid the collimated stellar streams that have been well-studied in prior work (such as Sgr, Orphan, GD1 and Pal 5-see, e.g., [14,43-45]) and instead focus on stellar structures that appear diffuse, amorphous, extended or "cloud-like" in nature, such as TriAnd, A13, and Mon/GASS. Initial interpretations of these morphologies suggested the structures could be shells — debris from the disruption of satellite galaxies on near-radial orbits—but as seen from an internal perspective [46]. However, our own and other recent observations instead suggest a common origin within the Galactic disk for stars associated with the low-latitude structures.

\subsection{Low-resolution Spectroscopy: Metallicities and Radial Velocities}

In our first study, we extended a prior sample of TriAnd M giants [27] by obtaining spectra of all candidate $\mathrm{M}$ giants identified by applying color-magnitude cuts to stars in the TriAnd region of the sky [16]. We identified M giants associated with the two proposed MSTO TriAnd structures (TriAnd 1 and 2, as named by [22,28]). M giant stars in both TriAnd 1 and 2 form clear over-densities in radial velocities with a small dispersion, $\sigma_{v} \approx 25 \mathrm{~km} \mathrm{~s}^{-1}$, compared to the background halo velocity distribution. The radial velocities of $\mathrm{M}$ giants in both TriAnd 1 and 2 follow the same sequence in velocity with a steady negative gradient of mean Galactic Standard-of-Rest (GSR) radial velocity $\left(v_{G S R}\right)$ with increasing Galactic longitude, $l$; see Figures 2 and 3, red triangles. We initially presented a dynamical model that simultaneously and approximately reproduces TriAnd 1 and 2 as tidal debris 
stripped over two separate pericentric passages from a single accreted satellite on a low-eccentricity, retrograde, near-planar orbit. The debris structures in the simulation were morphologically closer to streams than shells, but still subtended large areas on the sky as observed from the Sun's position. We note, however, that because of large distance uncertainties, the $M$ giants in TriAnd 1 and 2 are indistinguishable and overlap in distance, velocity distribution, and sky position; the existence of two distinct structures rather than a single extended structure has yet to be conclusively demonstrated (see [22] for some counter-arguments). Hereafter, we therefore refer to the TriAnd structures collectively, rather than individually.

In subsequent work, we continued this spectroscopic survey by observing M giant stars in A13 [30]. A13 overlaps the TriAnd clouds in Galactic longitude (but not latitude) at one end, and Mon/GASS at the other end, but is apparent in the Northern (rather than Southern) Galactic Hemisphere and at slightly brighter magnitudes than TriAnd. The spectra show that, like the TriAnd clouds, this structure has a coherent velocity structure with low dispersion and a steady gradient with longitude, $l$, confirming the genuine association of its members [15]; see Figure 2, orange squares.

Low-resolution spectroscopy has also been obtained for a sample of $\mathrm{M}$ giant stars that span $\approx 100^{\circ}$ of the Mon/GASS structure [26]. The candidate Mon/GASS member M giants also show a clear trend in GSR velocity with Galactic longitude, and appear to form a coherent sequence with both A13 and TriAnd; see Figure 2, yellow circles.

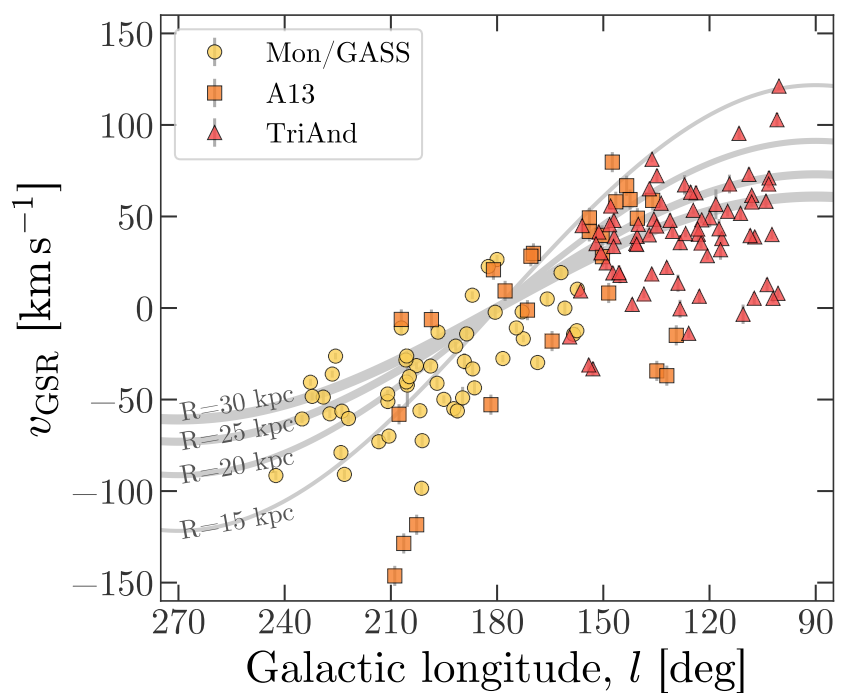

Figure 2. Summary of the velocity distribution of $M$ giants in each of the three low-latitude structures. Markers represent individual stars identified as likely members of each of the three structures discussed in this work (see figure legend). Grey curves show the expected $v_{\mathrm{GSR}}$ trends for circular orbits in the Galactic disk midplane with velocity equal to $220 \mathrm{~km} \mathrm{~s}^{-1}$ at several Galactocentric cylindrical radii, $R$, as indicated on the figure. Velocity uncertainties are typically the same as or smaller than the marker sizes. Reproduced from [15].

As mentioned above, Figure 2 (reproduced with data from previous work, [15]), summarizes the line-of-sight (GSR) velocity trends of $\mathrm{M}$ giants in each of the three low-latitude structures. Not only do these structures all have low dispersions $(\sim 25 \mathrm{~km} / \mathrm{s})$ relative to the stellar halo $(\sim 120 \mathrm{~km} / \mathrm{s})$ - which suggests that the structures themselves are real-they also appear to collectively exhibit a continuous gradient with Galactic longitude, $l$. This suggests that the structures may also be associated with one another, as part of a larger structure in the outer Galactic disk. 


\subsection{Stellar Populations and Other Kinematic Tracers}

Motivated by the observed, low-dispersion velocity distribution of the M giant stars in TriAnd, we sought to observe other distance tracer stars in the same region, determine their membership, and improve the distance estimates to the structure. We focused on and selected RR Lyrae stars in the TriAnd region from the Palomar Transient Factory (PTF; [47]), using a conservative distance cut to account for uncertainties in the RR Lyrae and M giant photometric distance estimates, $15 \mathrm{kpc}<d_{\odot}<35 \mathrm{kpc}$. We obtained spectra for $\approx 1 / 3$ of the total number of RR Lyrae in the M giant volume considered to be associated with TriAnd and measured radial velocities for these stars [41].

Figure 3 (reproduced from [41]) shows the results of our survey: unlike the M giants (triangles) the RR Lyrae stars (circles) show no clear, tight velocity sequence. By modeling both the RR Lyrae and $\mathrm{M}$ giants velocities as having been drawn from a mixture of two populations-one representing a low-dispersion sequence with varied dispersion, and one representing a halo population with large dispersion, both Gaussian - we showed that, after accounting for selection effects, the number ratio of RR Lyrae to M giants, $f_{\text {RR:MG }}$, within the overdensity is $f_{\text {RR:MG }}<0.38$ with $95 \%$ confidence.

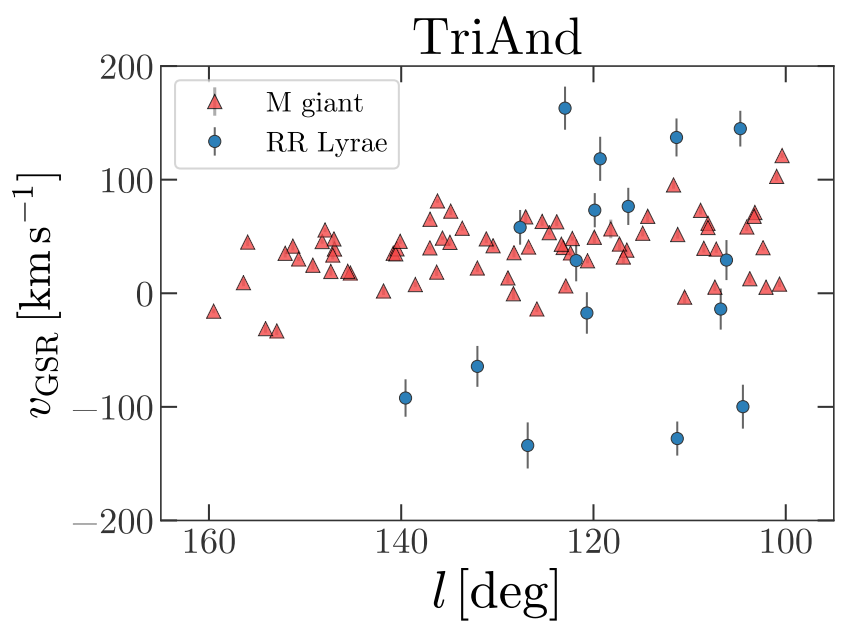

Figure 3. Comparison of the velocity distribution for $\mathrm{M}$ giants in the TriAnd structure (red triangles) with velocities for RR Lyrae stars in the same region of sky and distance range (blue circles). Radial velocity uncertainties of the $M$ giant stars are typically the size of the marker or smaller. Uncertainties for the RR Lyrae stars are shown with gray error bars. Note the low-dispersion sequence in the M giant velocities, unseen in the RR Lyrae star velocities, which look like typical halo stars with a large velocity dispersion. Reproduced from [41].

Since we were unable to find any RR Lyrae clearly associated with TriAnd, our attempt to measure more accurate distances to the structure was unsuccessful. However, the upper limit on the value of $f_{\mathrm{RR}: M G}$ was in itself interesting. RR Lyrae and M giants are tracers of populations with quite distinct metallicities: stars in the horizontal branch phase of evolution are typically only blue enough to cross the instability strip and become RR Lyrae if they have $[\mathrm{Fe} / \mathrm{H}] \lesssim-1.5$; and giant stars typically only evolve to colors red enough to become spectral class $M$ if they have $[\mathrm{Fe} / \mathrm{H}] \gtrsim-1.5$. Hence, a stellar population has to contain a significant range of metallicities to contain both types of stars. The metallicity distributions in nearly all existing satellite galaxies orbiting the Milky Way (e.g., [48]) are typically biased towards low metallicities (i.e., $[\mathrm{Fe} / \mathrm{H}]<-1$ ), such that they contain no or few $\mathrm{M}$ giant stars (i.e., $f_{\text {RR:MG }}=\infty$ ). The largest satellites (the Large Magellanic Cloud and Sgr) are exceptions as they contain substantial metal rich (i.e., $[\mathrm{Fe} / \mathrm{H}]>-1$ ) populations; these very metal-enriched dwarf galaxies have $f_{\text {RR:MG }} \sim 0.5$ in their still-bound stellar populations [41]. In contrast, the local Galactic disk is an overall metal-rich population and thus has very few RR Lyrae (i.e., $f_{\mathrm{RR}: M G} \sim 0$; [49]), more consistent with our findings for the stellar population of TriAnd. Moreover, results from the APOGEE 
survey show the outer disk populations have $[\mathrm{Fe} / \mathrm{H}]>-1[50]$, and hence it is also likely contain a very low fraction of RR Lyrae.

Our work on the stellar populations of the TriAnd region motivated us to look at possible associations of RR Lyrae with the M giant sequences found in Mon/GASS and A13. For the surveyed regions of these structures, we find $f_{R R: M G}$ values similar to those observed for TriAnd, and therefore consistent with membership of the Galactic disk (Sheffield et al., in prep.).

\subsection{High-Resolution Spectroscopy: Abundance Patterns}

The origin of stellar associations can also be explored through measurements of the detailed chemical abundances of their constituent stars. It is intriguing that our finding of the low-latitude structures all having stellar populations (as indicated by $f_{\mathrm{RR}: \mathrm{MG}}$ ) that look more like the disk than known Galactic satellites (Section 2.2) appears to be at odds with prior work on abundance patterns of stars in these structures. High-resolution spectra of $21 \mathrm{M}$ giants in Mon/GASS [39] showed [Ti/Fe] lower by up to 0.4 dex compared to the mean trends known for main-sequence stars of the Galactic disk (e.g., [51,52]) and a mean offset for $[\mathrm{Y} / \mathrm{Fe}]$ of about 0.2 dex at $[\mathrm{Fe} / \mathrm{H}] \approx-0.5$. A comparison with similar results for stars in Sgr [53] suggested that Mon/GASS may be more similar to Sgr than the disk, and therefore proposed an external origin for this structure. Subsequent work comes to similar conclusions for TriAnd stars [40].

To explore the apparent contradiction between the stellar populations and abundance work, we have recently obtained high-resolution $(R \sim 30,000-47,000)$ and high signal-to-noise $(>200$ per $\AA$ ) spectra of fifteen stars in the TriAnd and A13 overdensities. Fourteen stars were observed with the HIRES-S spectrograph at the Keck-1 telescope [62] and one star was observed using the UVES spectrograph at the VLT (Program ID: 097.B-0770A). Figure 4 shows the results of our preliminary analysis, with the average over all the stars in our sample presented as a black point. We find that the stars in these structures have a very narrow metallicity spread, with a value that is consistent with the prior metallicity estimates in TriAnd stars [40]. The TriAnd and A13 stars also have extremely similar chemical abundances to each other, with the abundance dispersion across the combined set of stars from both structures of $\leq 0.06$ dex for most chemical elements. The abundances of all measured $\alpha$-elements are uniformly enhanced at a level that is consistent with the abundances of the Milky Way disk stars (gray points; $[52,63,64]$ ). The $[\mathrm{Mg} / \mathrm{Fe}]$ value is also consistent with the measurements of $\alpha$-elements for the disk stars that have been found towards the outer disk in the APOGEE survey [50] (with the caveat that at a given [Fe/H] there is typically an offset of $\sim 0.2$ dex between $[\mathrm{Mg} / \mathrm{Fe}]$ measured in the infrared with APOGEE and other optical studies). The abundance ratios we derive are too high for the chemical abundance patterns observed in the stars of the Galactic satellites (colored points; [55,65-68]), which are known to have low, typically solar ([O/Fe]) or even sub-solar $([\mathrm{Mg} / \mathrm{Fe}],[\mathrm{Na} / \mathrm{Fe}])$, ratios at $[\mathrm{Fe} / \mathrm{H}] \sim-0.5$. However, note that very recent APOGEE results suggest elevated abundance levels for some elements are present in some stars in the Sgr dwarf at $[\mathrm{Fe} / \mathrm{H}] \lesssim-0.4$, so further work in this area is definitely warranted (Hasselquist et al., in prep.).

We are currently exploring one explanation for the disagreement between our and prior abundance work for stars in the same structures-that the differences in interpretation are due to differences in the observed datasets and spectroscopic modeling techniques. Past work $[39,40]$ analyzed a small wavelength region in the near-IR, limited to $150 \AA$ from 7440 to $7590 \AA$. Because of this limitation, they could include only $11 \mathrm{Fe}$ I and 2 Ti I lines in the determination of metallicities, and abundances. Our full analysis, through a much wider wavelength coverage and high SNR attained for the observed spectra, will allow us to include more then a hundred of Fe I and Fe II lines, as well as tens of Ti lines from both ionization stages (Ti II is important to check the influence of NLTE effects in Ti I). It has been shown that Ti I should be not be used in abundance studies because it is very sensitive to NLTE effects [69] (see also discussion in [70]). We are investigating the sensitivity of abundance diagnostics to the line selection and wavelength regimes by performing test computations on our own 
data, using a reduced line-list and comparing our $[\mathrm{Fe} / \mathrm{H}]$ and $[\mathrm{Ti} / \mathrm{Fe}]$ results between this and the analysis using the full line-list.
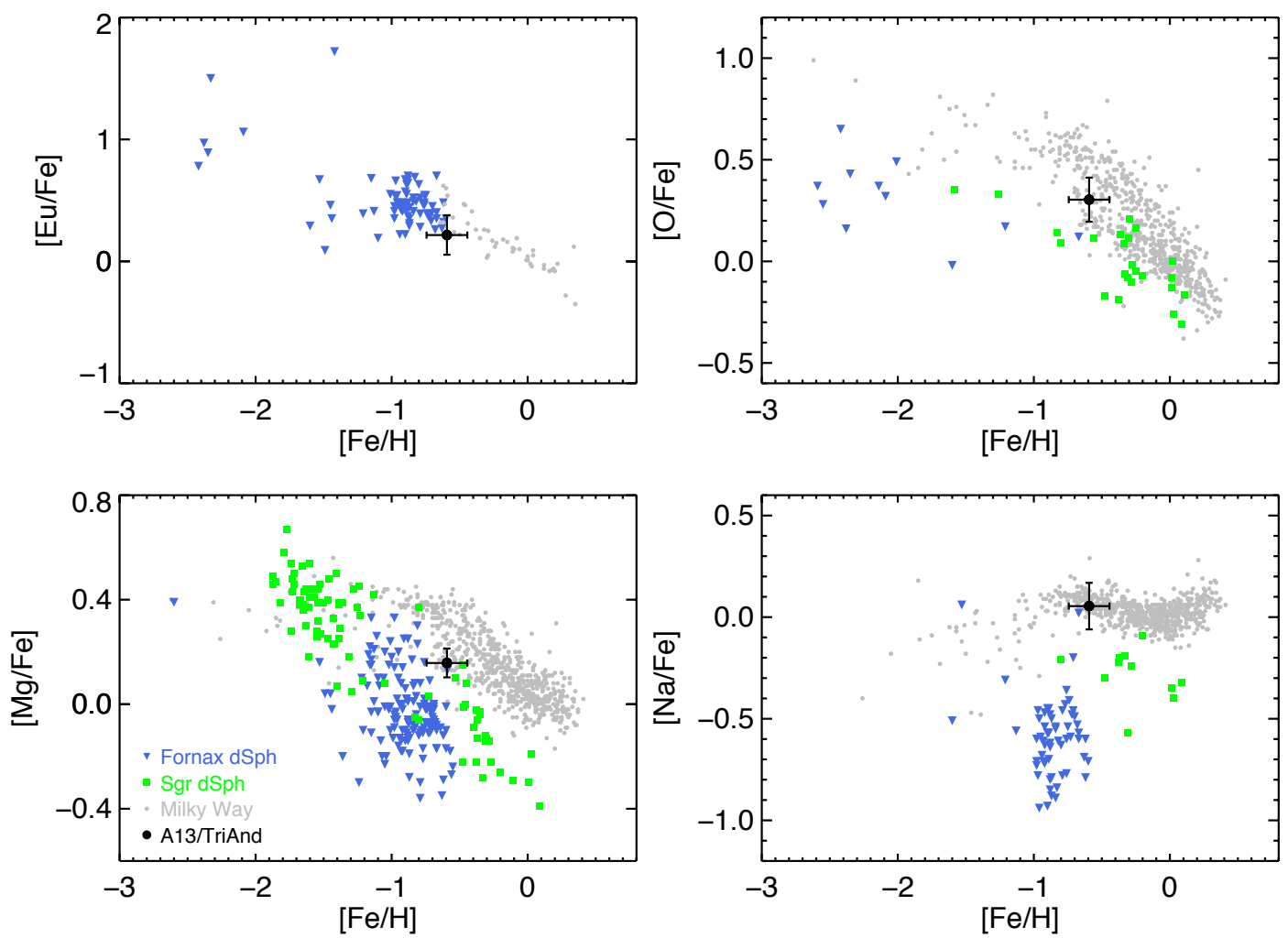

Figure 4. Abundance patterns for the thin and thick disk (grey symbols, from [52,54]), the Fornax (blue symbols, from [48,55-58]) and Sgr (green symbols, from [59-61]) dwarf spheroidal galaxies and our own results (black circle). The error bar on our measurement is the sum of the standard deviation of the stellar sample and a systematic error of the abundance measurement.

Overall, comparing to abundances of disk stars and satellite populations obtained using analogous data sets and reduction techniques, our current results indicate, in contrast to the prior work [39,40], that the birthplace of stars in TriAnd and A13 could be the outer Galactic disk (Bergemann et al., in prep.). The differences in our conclusions provide powerful motivation for a uniform survey of abundances for a much larger number of stars towards the Galactic anticenter and extending tens of kpc from the Sun (e.g., [50]), as well as at comparable metallicities in the few satellite galaxies large enough to host such metal-rich populations.

\subsection{Mapping Main-Sequence Stars in the Low-Latitude Structures}

While we have concentrated on follow-up studies of the known low-latitude structures as traced by M giants selected from 2MASS, knowledge of the spatial distribution of MSTO stars towards the anticenter region has been further refined using photometry from the PanSTARRS1 survey [21,25]. Recent studies using the SDSS [37] and Pan-STARRS1 (Lurie et al., in prep.) surveys employ the technique of subtracting color-magnitude diagrams (CMD's) derived from fields in their photometric data which were symmetrically placed at equal and opposite Galactic latitudes and at the same Galactic longitudes, analogous to that used for M giants in the original discovery paper for the TriAnd Clouds [27]. The vast number of MSTO stars allowed denser regions, closer to the Galactic plane and at smaller heliocentric distances to be explored. Both MSTO studies show overdense and clearly distinct 
arcs of stars oscillating between the Northern and Southern Hemispheres as the heliocentric distance was increased towards the anticenter of our Galaxy.

\subsection{Connecting the Low-Latitude Structures to Velocity Structure Near the Solar Radius}

Coincident with studies exploring structures at the very outer edge of our Galactic disk, large scale spectroscopic surveys have allowed a detailed re-examination of the local distribution of stellar velocities, within a few kpc of the Sun. Using data from the SDSS [71,72] and the RAdial Velocity Experiment (RAVE; $[73,74]$ ), asymmetries between the Northern and Southern Galactic Hemispheres have been seen in the density and velocity distributions of stars in the vicinity of the sun. Looking $\sim 2 \mathrm{kpc}$ out towards the Galactic anticenter, the Large Sky Area Multi-Object Fiber Spectroscopic Telescope (LAMOST; [75-77]) finds similar asymmetries in radial and vertical velocities [78]. The scale and sense of these asymmetries indicate moderate systematic motions (of order a few $\mathrm{km} / \mathrm{s}$ ) of stars within the disk perpendicular to the plane, suggesting both vertical movement of the midplane, and compression and rarefaction of the vertical scale (referred to as "bending" and "breathing" modes respectively-see, e.g., [79]). It is natural to associate these asymmetries in motion as a local manifestations of the oscillations traced in space over much larger scales [37,41].

\section{The Nature of Structures Around the Outer Disk-Summary of Theoretical Interpretations}

The observations summarized in Section 2 indicate that:

- $\quad$ the low-latitude structures-Mon/GASS, TriAnd, and A13 - each have low velocity dispersions supporting the genuine association of the candidate member stars;

- Mon/GASS, TriAnd, and A13 share a continuous sequence in mean GSR velocity as a function of Galactic longitude, suggestive of associations between these structures;

- the stellar populations in the structures (as indicated by $f_{\mathrm{RR}: \mathrm{MG}}$ ) are all more consistent with those in the Galactic disk rather than those observed in the stellar halo or Galactic satellites;

- the abundance patterns of stars in TriAnd and A13 are similar to those found in the disk of our Galaxy (although the discrepancy with prior work in this conclusion is still under investigation);

- the low-latitude structures (around the outer disk) may be connected to oscillating density and velocity structure on smaller scales, traced all the way back to the Solar neighborhood;

Taken together, we conclude that: (i) there is mounting evidence that Mon/GASS, TriAnd, and A13 represent populations of stars formed in the disk that now exist at extreme radii and heights above the Galactic disk; (ii) these structures are likely associated and part of a global system of vertical disk oscillations that can be traced all the way to the velocity asymmetries seen in the solar neighborhood; and (iii) the stellar populations in these structures are inconsistent with a picture in which they formed in a dwarf galaxy.

One natural interpretation of these collected observations is that the oscillations represent the response of the disk to an external perturbation-for example, the impact of a satellite galaxy that has been transmitted and amplified by its wake in the dark matter halo [38,80]. Prior work has already pointed to this as a possible explanation for the existence of Mon/GASS [34,35], with Sgr being pointed to as a plausible culprit for the perturbation [36]. It has also been demonstrated how perturbations from a satellite on an orbit perpendicular to the disk could lead to bending (at low relative impact velocity) and breathing (at higher relative velocity) modes that would be observed in the solar neighborhood as asymmetries in the local velocity distribution [79] and on larger scales as rings [81]. (Note that breathing modes can also be induced by non-axisymmetric features in the disk such as the bar and spiral arms; [82].) Simulations have also shown that Sgr could be responsible for local velocity structure [83]. Such interactions and corresponding disk features have been found to naturally occur in cosmological simulations [38].

Figure 5 illustrates these ideas with the results of simulations from our own recent work. Using simulations of a disk disturbed (separately) by satellites on orbits that mimic those expected 
for the Large Magellanic Cloud and Sgr [42], we extend the prior theoretical backdrop that looked at Mon/GASS to examine whether the extreme $(R, Z)$ locations of TriAnd stars could fit within the same picture. With different masses and orbits (and consequently different interaction strength, timings, and durations) these satellites necessarily induce distinct but overlapping signatures on the global structure of the disk. In more recent work, we found a model that was capable of reproducing the scales of the observed disturbances (radial wavelength and amplitude in space, as well as magnitude of offsets in velocity locally). The model required: the interaction of Sgr with the disk of the Milky Way to be followed for several passages longer than prior work (note that the length of Sgr's streams indicate that it has been impacting the disk for several pericentric passages e.g., [14]); Sgr to have sufficient initial mass and density to impact the disk in the last Gigayear with a remaining mass of $\sim 3 \times 10^{9} \mathrm{M}_{\odot}$; and the disk to be realized with stars existing as far out as $40 \mathrm{kpc}$ from the Galactic center to populate the regions corresponding to TriAnd. The interaction with the LMC modified the overall morphology of the structures induced, but was not sufficient alone to explain their properties. The full details of these results will be discussed in an upcoming paper (Laporte et al., in prep.).
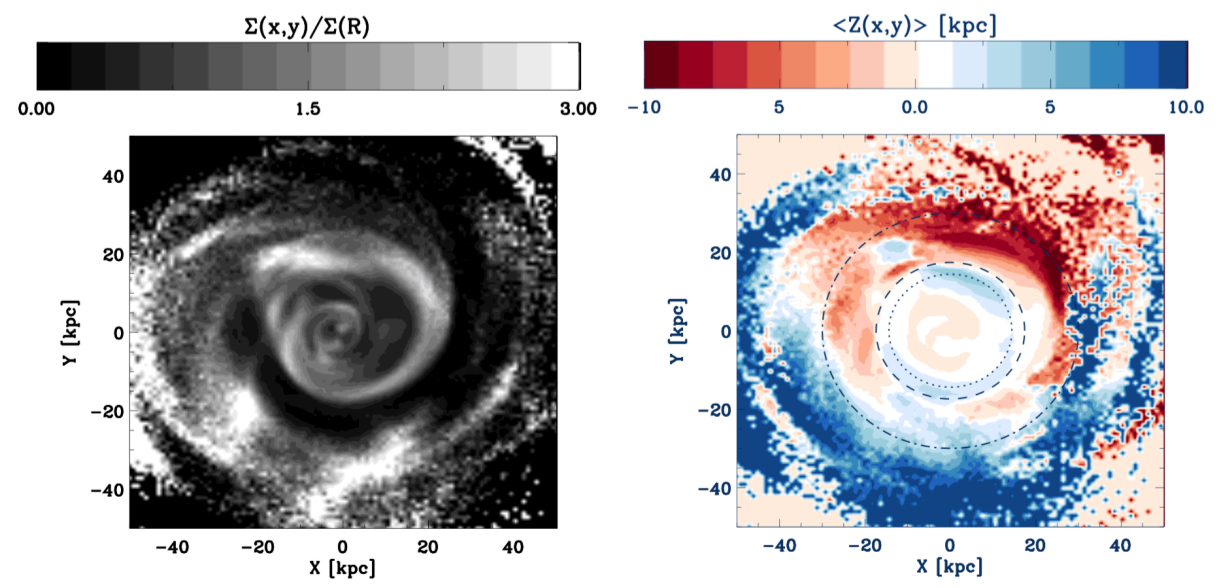

Figure 5. Visualizations at the end-point of a simulation of the impact of the Sgr dwarf galaxy on the Milky Way's disk from work currently in preparation (Laporte et al.). The simulation encompassed the last $\sim 6$ Gyrs of the interaction. Sgr had encountered the disk 6 times during this time and had a final mass of $\sim 3 \times 10^{9} M_{\circ}$. The left hand panel shows density fluctuations around the mean at each annulus in the disk. The right hand panel shows the average positions of particles above/below the disk at each point. Note that material is kicked to as much as $10 \mathrm{kpc}$ from the midplane at distances of 20-30 kpc from the Galactic center.

\section{Discussion-Observational Prospects}

\subsection{The Milky Way}

While the connections that have already been made between the different low-latitude structures and the disk population are convincing, there are several possible directions for further observations to strengthen these claims. Kinematic and abundance information and density measurements for more tracers and over a larger volume would greatly facilitate an informative comparison to theoretical work, allowing more detailed interpretations of the history of our Galaxy.

The most obvious direction is to obtain proper motions and more accurate distance estimates to the known features, using the candidate members discussed in this work to search for other tracers. For example, proper motions for the "Anti-Center Stream" (ACS) (a thin, coherent density structure, which may or may not be part of the larger Mon/GASS system; [84]) indicate that stars in the ACS are not actually moving along the spatial extent of the stream [85]. This is inconsistent with expectations for the behavior of debris from a destroyed satellite. If similar measurements of proper motions of stars in all of the low-latitude structures showed significant motion perpendicular to the Galactic disk, 
this would provide even more evidence of a disk origin and connection to local oscillations (first results are just being reported in this region [86]). With precise proper motions, the velocity information would also place important constraints on dynamical models of the disk (see Section 5). In upcoming data-releases, astrometric measurements from the Gaia mission [87] are poised to provide these data. Expected proper motion uncertainties for the closest $M$ giant stars ( $\approx 5 \mathrm{kpc})$ in Mon/GASS correspond to tangential velocity uncertainties of $\approx 1-2 \mathrm{~km} \mathrm{~s}^{-1}$ for M giants with tangential velocities $<50 \mathrm{~km} \mathrm{~s}^{-1}$. For the farthest known M giant stars in TriAnd $(\approx 35 \mathrm{kpc})$, tangential velocity uncertainties will be $\approx 7-12 \mathrm{~km} \mathrm{~s}^{-1}$ for $\mathrm{M}$ giants with tangential velocities $<50 \mathrm{~km} \mathrm{~s}^{-1}$.

The next decade will see first light for the Large Synoptic Survey Telescope (LSST; [88]), which will survey the sky to the same depth as SDSS every three days. Over time, these data can be combined to detect MSTO stars out to $100 \mathrm{kpc}$. Hence the exquisite maps from SDSS and PanSTARRS that exploited the dense coverage of this tracer to reveal disk oscillations out to Mon/GASS can be extended to A13 and TriAnd and beyond.

Another complimentary direction for future data is to extend spectroscopic maps to fainter magnitudes and global scales. The Gaia catalogue will soon be enhanced by surveys from several ground-based, wide-field, multi-object spectrographs on larger telescopes capable of reaching to fainter magnitudes (e.g., WEAVE on the William Herschel Telescope, 4MOST and the Prime Focus Spectrograph on the Subaru telescope-[89-91]). However, the reach of these surveys towards lower latitudes all the way to the disk plane will be limited by extinction. An infrared spectroscopic survey, such as APOGEE [92] , could overcome this limitation. APOGEE is capable of building catalogues of radial velocities and abundance patterns for 15 chemical species across the Galactic disk, bulge, bar, halo, star clusters, and dwarf galaxies - the type of homogeneous data set needed to compare the low latitude structures to other Galactic components.

\subsection{Other Galaxies}

TriAnd is estimated to have a surface brightness below $32 \mathrm{mag} / \operatorname{arcsec}^{2}$ [93], so studying similar features around other galaxies presents a significant challenge. Nevertheless, there are growing samples of galaxies within and beyond the Local Group being mapped to depths close to this target.

For nearby galaxies, these levels are approached through star counts studies, most spectacularly for the case of our nearest neighbor, the Andromeda Galaxy, where giant star counts have revealed a significantly extended and richly featured outer stellar disk [94,95]. Analogous studies have been carried out for galaxies up to distances of several Mpc (e.g., [96,97]), although the focus of these studies has typically been on detecting the stellar halos of these objects. The great advantage of star-count studies is the ability to reach extremely low surface brightnesses-depths below $30 \mathrm{mag} / \mathrm{arcsec}^{2} \mathrm{have}^{-}$ been estimated for the density profile of M31's and other galaxies' stellar halos [98,99].

Star count studies are limited to the galaxies in the volume within which stars can be resolved. Several dedicated surveys have made innovative advances in studying galaxies to low surface-brightness using a variety of techniques to reach limits below $30 \mathrm{mag} / \mathrm{arcsec}^{2}$ in integrated light (e.g., [100-103]), although these studies face the twin challenges of calibration and Galactic cirrus to overcome.

Looking to the future, NASA's proposed Wide Field InfraRed Survey Telescope (WFIRST), with its wide field of view and high resolution, offers the possibility of extending the deep star-count sensitivities now achieved in MW and M31 to all galaxies within 10 Mpc [104]. In the next decade, images in integrated light from LSST can be combined to be sensitive to slightly shallower depth ( $29 \mathrm{mag} / \mathrm{arcsec}^{2}$, see [88]), but for vast numbers (many millions!) of galaxies.

\section{Conclusions-What Might These Structures Tell Us about Galaxies?}

The above sections summarize observational evidence for large scale vertical oscillations of the Galactic plane present in the Solar neighborhood and reaching out beyond the traditional edge of our stellar disk. We have also discussed theoretical studies that suggest that these oscillations could 
be caused by, and contain the signatures of, ongoing interactions of the Milky Way with its satellite system. Moreover, there are numerous observational prospects for extending this work both to map the Milky Way globally and to look for analogous features around many other galaxies.

Now that we have a physical picture of the origin of such planar undulations, as well as prospects for mapping them further within the Milky Way and detecting analogous substructures around other galaxies, we can move on to discussing how useful they are for constraining the dynamics and evolution of galaxies. While the mere existence of these low surface brightness structures around the outskirts of galaxies is interesting, they contain only a tiny fraction of the stars in galaxies and these are spread out over a large area-these properties naturally make such structures difficult to map, either because their unique signatures can be lost in the foreground star counts (e.g., in the Milky Way), or because the required surface brightness limits for detection are prohibitively low (for integrated light).

Conversely, these features may prove to be particularly powerful probes of interactions and histories, precisely because they contain so little mass: they can be modeled as test particles responding to an external perturbation.

Below are just three examples of where these structures could promise new insights into some classic questions in galactic astronomy.

- Disk heating mechanisms - It has been understood for a long time that disks can evolve significantly due to mergers, major or minor, and hence that their current structures bear witness to their accretion history [105-108]. This understanding has fueled a significant literature on simulations investigating the importance of the heating of galactic disks in response to encounters with other dark matter halos (that may or may not contain their own galaxies) [109-118]. In general, these studies have concentrated on the overall effects of many encounters on global properties, such as the thickness and vertical velocity dispersion in disks. The results of these simulations have traditionally been compared to the spatial and velocity scales in samples of galaxies. In contrast, the identification and mapping of vertical waves associated with ongoing interactions in the Milky Way gives us the opportunity to dissect individual disk heating events in progress (e.g., the impact of Sgr). We can use this to check our understanding of the mechanism directly and in detail rather than assessing its importance through collective effects and longterm, phase-mixed signatures.

- Stellar halo formation processes - The last decade has seen increasing interest in assessing how much of the content of our stellar halo could be made "in situ" along with other components of the Milky Way rather than accreted from other objects. Hydrodynamical simulations of galaxy formation suggest that tens of percent of the stars in the inner halo might be formed "in situ", either along their current orbits or "kicked-out" from orbits that were originally in the disk [119-127]. Preliminary arguments for the existence of an "in situ" population were based on transitions in the density or orbital structures of stellar halos (e.g., [128]). However, such transitions were also found to occur naturally in purely-accreted models of stellar halos [129]. More convincing observational evidence for stars in the halo that have been "kicked-out" of the disk is just beginning to emerge through studies that look for stars with halo-like kinematics, but disk-like abundances around M31 [130] and the Milky Way [70,131,132]. The work outlined above in Sections 2 and 3 adds new perspectives on the "kicked-out-disk" stellar halo formation process with the detection and modeling of disk stars that may be in transition from the disk to the halo.

- Galactoseismic probes of interactions and dark matter - The response of a disk to an encounter will depend on its own properties, the properties of the dark matter halo in which it is embedded, and the mass and orbit of the perturbing satellite. This leads to the suggestion that, analogous to helioseismic investigations of the structure of our Sun, maps of a disk response-such as those described in Section 2 for our Milky Way—-might be similarly inverted to tell us about, for example, the structure of the dark matter halo [71]. Indeed, recent investigations into the signatures of encounters in the very outskirts of extended HI disks have successfully used simulations combined 
with an analytic understanding to find how the observed characteristics of the disturbed gas can be simply related to properties of the perturbing object [133-135].

Overall, the breadth and depth of studies summarized in this Article are indicative of the developing interest in the new discipline of Galactoseismology, fueled by the imminence of another leap in the scale of available data sets. The remaining challenge is to build the models and appropriate tools to compare to obervations that can take full advantage of these opportunities to significantly advance our understanding of galactic dynamics and evolution.

Acknowledgments: Much of the work reviewed in this paper was made possible by NSF grant AST-1312196. Kathryn V. Johnston was supported by NSF grant AST-1614743 while writing the review.

Author Contributions: This paper summarizes results from the team of listed authors. It was written by Kathryn V. Johnston and Adrian M. Price-Whelan, with section contributions from Maria Bergemann Figures were made by Adrian M. Price-Whelan (using data consolidated by Ting S. Li) and Chervin Laporte All the authors reviewed and commented on the drafts.

Conflicts of Interest: The authors declare no conflict of interest.

\section{References}

1. European Space Agency (ESA). (Ed.) The HIPPARCOS and TYCHO Catalogues. Astrometric and Photometric Star Catalogues Derived from the ESA HIPPARCOS Space Astrometry Mission; ESA Special Publication; ESA Publications Division: Noordwijk, The Netherland, 1997; Volume 1200.

2. Dehnen, W. The Distribution of Nearby Stars in Velocity Space Inferred from HIPPARCOS Data. Astron. J. 1998, 115, 2384-2396.

3. Dehnen, W. The Effect of the Outer Lindblad Resonance of the Galactic Bar on the Local Stellar Velocity Distribution. Astron. J. 2000, 119, 800-812.

4. York, D.G.; Adelman, J.; Anderson, J.E., Jr.; Anderson, S.F.; Annis, J.; Bahcall, N.A.; Bakken, J.A.; Barkhouser, R.; Bastian, S.; Berman, E.; et al. The Sloan Digital Sky Survey: Technical Summary. Astron. J. 2000, 120, 1579.

5. Stoughton, C.; Lupton, R.H.; Bernardi, M.; Blanton, M.R.; Burles, S.; Castander, F.J.; Connolly, A.J.; Eisenstein, D.J.; Frieman, J.A.; Hennessy, G.S.; et al. Sloan Digital Sky Survey: Early Data Release. Astron. J. 2002, 123, 485-548.

6. Abazajian, K.; Adelman-McCarthy, J.K.; Agüeros, M.A.; Allam, S.S.; Anderson, S.F.; Annis, J.; Bahcall, N.A.; Baldry, I.K.; Bastian, S.; Berlind, A.; et al. The First Data Release of the Sloan Digital Sky Survey. Astron. J. 2003, 126, 2081-2086.

7. Newberg, H.J.; Yanny, B.; Rockosi, C.; Grebel, E.K.; Rix, H.W.; Brinkmann, J.; Csabai, I.; Hennessy, G.; Hindsley, R.B.; Ibata, R.; et al. The Ghost of Sagittarius and Lumps in the Halo of the Milky Way. Astrophys. J. 2002, 569, 245-274.

8. Belokurov, V.; Zucker, D.B.; Evans, N.W.; Gilmore, G.; Vidrih, S.; Bramich, D.M.; Newberg, H.J.; Wyse, R.F.G.; Irwin, M.J.; Fellhauer, M.; et al. The Field of Streams: Sagittarius and Its Siblings. Astrophys. J. 2006, 642, L137-L140.

9. Bullock, J.S.; Kravtsov, A.V.; Weinberg, D.H. Hierarchical Galaxy Formation and Substructure in the Galaxy's Stellar Halo. Astrophys. J. 2001, 548, 33-46.

10. Bullock, J.S.; Johnston, K.V. Tracing Galaxy Formation with Stellar Halos. I. Methods. Astrophys. J. 2005, 635, 931-949.

11. Bell, E.F.; Zucker, D.B.; Belokurov, V.; Sharma, S.; Johnston, K.V.; Bullock, J.S.; Hogg, D.W.; Jahnke, K.; de Jong, J.T.A.; Beers, T.C.; et al. The Accretion Origin of the Milky Way's Stellar Halo. Astrophys. J. 2008, 680, 295-311.

12. Nikolaev, S.; Weinberg, M.D.; Skrutskie, M.F.; Cutri, R.M.; Wheelock, S.L.; Gizis, J.E.; Howard, E.M. A Global Photometric Analysis of 2MASS Calibration Data. Astrophys. J. 2000, 120, 3340-3350.

13. Majewski, S.R.; Skrutskie, M.F.; Weinberg, M.D.; Ostheimer, J.C. A Two Micron All Sky Survey View of the Sagittarius Dwarf Galaxy. I. Morphology of the Sagittarius Core and Tidal Arms. Astrophys. J. 2003, 599, 1082-1115. 
14. Law, D.R.; Majewski, S.R. The Sagittarius Dwarf Galaxy: A Model for Evolution in a Triaxial Milky Way Halo. Astrophys. J. 2010, 714, 229-254.

15. Li, T.S.; Sheffield, A.A.; Johnston, K.V.; Marshall, J.L.; Majewski, S.R.; Price-Whelan, A.M.; Damke, G.J.; Beaton, R.L.; Richardson, W.; Sharma, S.; et al. Exploring Halo Substructure with Giant Stars. XV. Discovery of a Connection between the Monoceros Ring and the Triangulum-Andromeda Overdensity? Astrophys. J. 2017, 844, doi:10.3847/1538-4357/aa7a0d.

16. Sheffield, A.A.; Johnston, K.V.; Majewski, S.R.; Damke, G.; Richardson, W.; Beaton, R.; Rocha-Pinto, H.J. Exploring Halo Substructure with Giant Stars. XIV. The Nature of the Triangulum-Andromeda Stellar Features. Astrophys. J. 2014, 793, doi:10.1088/0004-637X/793/1/62.

17. Yanny, B.; Newberg, H.J.; Grebel, E.K.; Kent, S.; Odenkirchen, M.; Rockosi, C.M.; Schlegel, D.; Subbarao, M.; Brinkmann, J.; Fukugita, M.; et al. A Low-Latitude Halo Stream around the Milky Way. Astrophys. J. 2003, 588, 824-841.

18. Ibata, R.A.; Irwin, M.J.; Lewis, G.F.; Ferguson, A.M.N.; Tanvir, N. One ring to encompass them all: A giant stellar structure that surrounds the Galaxy. Mon. Not. R. Astron. Soc. 2003, 340, L21-L27.

19. Grillmair, C.J. Substructure in Tidal Streams: Tributaries in the Anticenter Stream. Astrophys. J. Lett. 2006, 651, L29-L32.

20. Grillmair, C.J.; Carlin, J.L.; Majewski, S.R. Fishing in Tidal Streams: New Radial Velocity and Proper Motion Constraints on the Orbit of the Anticenter Stream. Astrophys. J. Lett. 2008, 689, L117-L120.

21. Slater, C.T.; Bell, E.F.; Schlafly, E.F.; Morganson, E.; Martin, N.F.; Rix, H.W.; Peñarrubia, J.; Bernard, E.J.; Ferguson, A.M.N.; Martinez-Delgado, D.; et al. The Complex Structure of Stars in the Outer Galactic Disk as Revealed by Pan-STARRS1. Astrophys. J. 2014, 791, 9, doi:10.1088/0004-637X/791/1/9.

22. Martin, N.F.; Ibata, R.A.; Rich, R.M.; Collins, M.L.M.; Fardal, M.A.; Irwin, M.J.; Lewis, G.F.; McConnachie, A.W.; Babul, A.; Bate, N.F.; et al. The PAndAS Field of Streams: Stellar Structures in the Milky Way Halo toward Andromeda and Triangulum. Astrophys. J. 2014, 787, doi:10.1088/0004-637X/787/1/19.

23. Deason, A.J.; Belokurov, V.; Hamren, K.M.; Koposov, S.E.; Gilbert, K.M.; Beaton, R.L.; Dorman, C.E.; Guhathakurta, P.; Majewski, S.R.; Cunningham, E.C. TriAnd and its siblings: Satellites of satellites in the Milky Way halo. Mon. Not. R. Astron. Soc. 2014, 444, 3975-3985.

24. Robin, A.C.; Creze, M.; Mohan, V. The edge of the Galactic disk. Astrophys. J. Lett. 1992, 400, L25-L27.

25. Morganson, E.; Conn, B.; Rix, H.W.; Bell, E.F.; Burgett, W.S.; Chambers, K.; Dolphin, A.; Draper, P.W.; Flewelling, H.; Hodapp, K.; et al. Mapping the Monoceros Ring in 3D with Pan-STARRS1. Astrophys. J. 2016, 825, doi:10.3847/0004-637X/825/2/140.

26. Crane, J.D.; Majewski, S.R.; Rocha-Pinto, H.J.; Frinchaboy, P.M.; Skrutskie, M.F.; Law, D.R. Exploring Halo Substructure with Giant Stars: Spectroscopy of Stars in the Galactic Anticenter Stellar Structure. Astrophys. J. Lett. 2003, 594, L119-L122.

27. Rocha-Pinto, H.J.; Majewski, S.R.; Skrutskie, M.F.; Crane, J.D.; Patterson, R.J. Exploring Halo Substructure with Giant Stars: A Diffuse Star Cloud or Tidal Debris around the Milky Way in Triangulum-Andromeda. Astrophys. J. 2004, 615, 732-737.

28. Martin, N.F.; Ibata, R.A.; Irwin, M. Galactic Halo Stellar Structures in the Triangulum-Andromeda Region. Astrophys. J. Lett. 2007, 668, L123-L126.

29. Sharma, S.; Johnston, K.V. A Group Finding Algorithm for Multidimensional Data Sets. Astrophys. J. 2009, 703, 1061-1077.

30. Sharma, S.; Johnston, K.V.; Majewski, S.R.; Muñoz, R.R.; Carlberg, J.K.; Bullock, J. Group Finding in the Stellar Halo Using M-giants in the Two Micron All Sky Survey: An Extended View of the Pisces Overdensity? Astrophys. J. 2010, 722, 750-759.

31. Peñarrubia, J.; Martínez-Delgado, D.; Rix, H.W.; Gómez-Flechoso, M.A.; Munn, J.; Newberg, H.; Bell, E.F.; Yanny, B.; Zucker, D.; Grebel, E.K. A Comprehensive Model for the Monoceros Tidal Stream. Astrophys. J. 2005, 626, 128-144.

32. Momany, Y.; Zaggia, S.R.; Bonifacio, P.; Piotto, G.; De Angeli, F.; Bedin, L.R.; Carraro, G. Probing the Canis Major stellar over-density as due to the Galactic warp. Astron. Astrophys. 2004, 421, L29-L32.

33. Momany, Y.; Zaggia, S.; Gilmore, G.; Piotto, G.; Carraro, G.; Bedin, L.R.; de Angeli, F. Outer structure of the Galactic warp and flare: explaining the Canis Major over-density. Astron. Astrophys. 2006, 451, 515-538. 
34. Kazantzidis, S.; Bullock, J.S.; Zentner, A.R.; Kravtsov, A.V.; Moustakas, L.A. Cold Dark Matter Substructure and Galactic Disks. I. Morphological Signatures of Hierarchical Satellite Accretion. Astrophys. J. 2008, $688,254-276$.

35. Younger, J.D.; Besla, G.; Cox, T.J.; Hernquist, L.; Robertson, B.; Willman, B. On the Origin of Dynamically Cold Rings around the Milky Way. Astrophys. J. Lett. 2008, 676, doi:10.1086/587099.

36. Purcell, C.W.; Bullock, J.S.; Tollerud, E.J.; Rocha, M.; Chakrabarti, S. The Sagittarius impact as an architect of spirality and outer rings in the Milky Way. Nature 2011, 477, 301-303.

37. Xu, Y.; Newberg, H.J.; Carlin, J.L.; Liu, C.; Deng, L.; Li, J.; Schönrich, R.; Yanny, B. Rings and Radial Waves in the Disk of the Milky Way. Astrophys. J. 2015, 801, doi:10.1088/0004-637X/801/2/105.

38. Gómez, F.A.; White, S.D.M.; Marinacci, F.; Slater, C.T.; Grand, R.J.J.; Springel, V.; Pakmor, R. A fully cosmological model of a Monoceros-like ring. Mon. Not. R. Astron. Soc. 2016, 456, 2779-2793.

39. Chou, M.Y.; Majewski, S.R.; Cunha, K.; Smith, V.V.; Patterson, R.J.; Martínez-Delgado, D. The Chemical Evolution of the Monoceros Ring/Galactic Anticenter Stellar Structure. Astrophys. J. Lett. 2010, 720, L5-L10.

40. Chou, M.Y.; Majewski, S.R.; Cunha, K.; Smith, V.V.; Patterson, R.J.; Martínez-Delgado, D. First Chemical Analysis of Stars in the Triangulum-Andromeda Star Cloud. Astrophys. J. Lett. 2011, 731, doi:10.1088/ 2041-8205/731/2/L30.

41. Price-Whelan, A.M.; Johnston, K.V.; Sheffield, A.A.; Laporte, C.F.P.; Sesar, B. A reinterpretation of the Triangulum-Andromeda stellar clouds: A population of halo stars kicked out of the Galactic disc. Mon. Not. R. Astron. Soc. 2015, 452, 676-685.

42. Laporte, C.F.P.; Gómez, F.A.; Besla, G.; Johnston, K.V.; Garavito-Camargo, N. Response of the Milky Way's disc to the Large Magellanic Cloud in a first infall scenario. Mon. Not. R. Astron. Soc. 2016, 1-13.

43. Koposov, S.E.; Rix, H.W.; Hogg, D.W. Constraining the Milky Way Potential with a Six-Dimensional Phase-Space Map of the GD-1 Stellar Stream. Astrophys. J. 2010, 712, 260-273.

44. Küpper, A.H.W.; Balbinot, E.; Bonaca, A.; Johnston, K.V.; Hogg, D.W.; Kroupa, P.; Santiago, B.X. Globular Cluster Streams as Galactic High-Precision Scales: The Poster Child Palomar 5. Astrophys. J. 2015, 803, doi:10.1088/0004-637X/803/2/80.

45. Bovy, J.; Bahmanyar, A.; Fritz, T.K.; Kallivayalil, N. The Shape of the Inner Milky Way Halo from Observations of the Pal 5 and GD-1 Stellar Streams. Astrophys. J. 2016, 833, doi:10.3847/1538-4357/833/1/31.

46. Johnston, K.V.; Bullock, J.S.; Sharma, S.; Font, A.; Robertson, B.E.; Leitner, S.N. Tracing Galaxy Formation with Stellar Halos. II. Relating Substructure in Phase and Abundance Space to Accretion Histories. Astrophys. J. 2008, 689, 936-957.

47. Law, N.M.; Kulkarni, S.R.; Dekany, R.G.; Ofek, E.O.; Quimby, R.M.; Nugent, P.E.; Surace, J.; Grillmair, C.C.; Bloom, J.S.; Kasliwal, M.M.; et al. The Palomar Transient Factory: System Overview, Performance, and First Results. Publ. Astron. Soc. Pac. 2009, 121, doi:10.1086/648598.

48. Kirby, E.N.; Lanfranchi, G.A.; Simon, J.D.; Cohen, J.G.; Guhathakurta, P. Multi-element Abundance Measurements from Medium-resolution Spectra. III. Metallicity Distributions of Milky Way Dwarf Satellite Galaxies. Astrophys. J. 2011, 727, doi:10.1088/0004-637X/727/2/78 .

49. Amrose, S.; Mckay, T. A Calculation of the Mean Local RR Lyrae Space Density Using ROTSE. Astrophys. J. Lett. 2001, 560, L151-L154.

50. Hayden, M.R.; Bovy, J.; Holtzman, J.A.; Nidever, D.L.; Bird, J.C.; Weinberg, D.H.; Andrews, B.H.; Majewski, S.R.; Allende Prieto, C.; Anders, F.; et al. Chemical Cartography with APOGEE: Metallicity Distribution Functions and the Chemical Structure of the Milky Way Disk. Astrophys. J. 2015, 808, doi:10.1088/0004-637X/808/2/132.

51. Reddy, B.E.; Tomkin, J.; Lambert, D.L.; Allende Prieto, C. The chemical compositions of Galactic disc F and G dwarfs. Mon. Not. R. Astron. Soc. 2003, 340, 304-340.

52. Bensby, T.; Feltzing, S.; Oey, M.S. Exploring the Milky Way stellar disk. A detailed elemental abundance study of $714 \mathrm{~F}$ and G dwarf stars in the solar neighbourhood. Astron. Astrophys. 2014, 562, doi:10.1051/0004-6361/201322631.

53. Chou, M.Y.; Cunha, K.; Majewski, S.R.; Smith, V.V.; Patterson, R.J.; Martínez-Delgado, D.; Geisler, D. A Two Micron All Sky Survey View of the Sagittarius Dwarf Galaxy. VI. s-Process and Titanium Abundance Variations Along the Sagittarius Stream. Astrophys. J. 2010, 708, 1290-1309.

54. Battistini, C.; Bensby, T. The origin and evolution of $r$ - and s-process elements in the Milky Way stellar disk. Astron. Astrophys. 2016, 586, doi:10.1051/0004-6361/201527385. 
55. Shetrone, M.; Venn, K.A.; Tolstoy, E.; Primas, F.; Hill, V.; Kaufer, A. VLT/UVES Abundances in Four Nearby Dwarf Spheroidal Galaxies. I. Nucleosynthesis and Abundance Ratios. Astrophys. J. 2003, 125, 684-706.

56. Letarte, B.; Hill, V.; Jablonka, P.; Tolstoy, E.; François, P.; Meylan, G. VLT/UVES spectroscopy of individual stars in three globular clusters in the Fornax dwarf spheroidal galaxy. Astron. Astrophys. 2006, 453, 547-554.

57. Letarte, B.; Hill, V.; Tolstoy, E.; Jablonka, P.; Shetrone, M.; Venn, K.A.; Spite, M.; Irwin, M.J.; Battaglia, G.; Helmi, A.; et al. A high-resolution VLT/FLAMES study of individual stars in the centre of the Fornax dwarf spheroidal galaxy. Astron. Astrophys. 2010, 523, doi:10.1051/0004-6361/200913413.

58. Larsen, S.S.; Brodie, J.P.; Strader, J. Detailed abundance analysis from integrated high-dispersion spectroscopy: Globular clusters in the Fornax dwarf spheroidal. Astron. Astrophys. 2012, 546, doi:10.1051/ 0004-6361/201219895.

59. McWilliam, A.; Smecker-Hane, T.A. The Composition of the Sagittarius Dwarf Spheroidal Galaxy, and Implications for Nucleosynthesis and Chemical Evolution. In Astronomical Society of the Pacific Conference Series, Proceedings of the Cosmic Abundances as Records of Stellar Evolution and Nucleosynthesis, Austin, TX, USA, 17-19 June 2004; Barnes, T.G., III, Bash, F.N., Eds.; Springer: Berlin/Heidelberg, Germany, 2005; Volume 336; p. 221.

60. Sbordone, L.; Bonifacio, P.; Buonanno, R.; Marconi, G.; Monaco, L.; Zaggia, S. The exotic chemical composition of the Sagittarius dwarf spheroidal galaxy. Astron. Astrophys. 2007, 465, 815-824.

61. Mucciarelli, A.; Bellazzini, M.; Ibata, R.; Romano, D.; Chapman, S.C.; Monaco, L. Chemical abundances in the nucleus of the Sagittarius dwarf spheroidal galaxy. Astron. Astrophys. 2017, doi:10.1051/00046361/201730707.

62. Vogt, S.S.; Allen, S.L.; Bigelow, B.C.; Bresee, L.; Brown, B.; Cantrall, T.; Conrad, A.; Couture, M.; Delaney, C.; Epps, H.W.; et al. HIRES: The high-resolution echelle spectrometer on the Keck 10-m Telescope. In Instrumentation in Astronomy VIII; Crawford, D.L., Craine, E.R., Eds.; SPIE: Bellingham, WA, USA, 1994; Volume 2198, Proc. SPIE, p. 362.

63. Fuhrmann, K. Nearby stars of the Galactic disk and halo. III. Astron. Nachr. 2004, 325, 3-80.

64. Bergemann, M.; Ruchti, G.R.; Serenelli, A.; Feltzing, S.; Alves-Brito, A.; Asplund, M.; Bensby, T.; Gruyters, P.; Heiter, U.; Hourihane, A.; et al. The Gaia-ESO Survey: Radial metallicity gradients and age-metallicity relation of stars in the Milky Way disk. Astron. Astrophys. 2014, 565, doi:10.1051/0004-6361/201423456.

65. Bonifacio, P.; Hill, V.; Molaro, P.; Pasquini, L.; Di Marcantonio, P.; Santin, P. First results of UVES at VLT: Abundances in the Sgr dSph. Astron. Astrophys. 2000, 359, 663-668.

66. Shetrone, M.D.; Côté, P.; Sargent, W.L.W. Abundance Patterns in the Draco, Sextans, and Ursa Minor Dwarf Spheroidal Galaxies. Astrophys. J. 2001, 548, 592-608.

67. Tolstoy, E.; Hill, V.; Tosi, M. Star-Formation Histories, Abundances, and Kinematics of Dwarf Galaxies in the Local Group. Ann. Rev. Astron. Astrophys. 2009, 47, 371-425.

68. De Boer, T.J.L.; Tolstoy, E.; Lemasle, B.; Saha, A.; Olszewski, E.W.; Mateo, M.; Irwin, M.J.; Battaglia, G. The episodic star formation history of the Carina dwarf spheroidal galaxy. Astron. Astrophys. 2014, 572, doi:10.1051/0004-6361/201424119.

69. Bergemann, M. Ionization balance of Ti in the photospheres of the Sun and four late-type stars. Mon. Not. R. Astron. Soc. 2011, 413, 2184-2198.

70. Sheffield, A.A.; Majewski, S.R.; Johnston, K.V.; Cunha, K.; Smith, V.V.; Cheung, A.M.; Hampton, C.M.; David, T.J.; Wagner-Kaiser, R.; Johnson, M.C.; et al. Identifying Contributions to the Stellar Halo from Accreted, Kicked-out, and In Situ Populations. Astrophys. J. 2012, 761, doi:10.1088/0004-637X/761/2/161.

71. Widrow, L.M.; Gardner, S.; Yanny, B.; Dodelson, S.; Chen, H.Y. Galactoseismology: Discovery of Vertical Waves in the Galactic Disk. Astrophys. J. Lett. 2012, 750, doi:10.1088/2041-8205/750/2/L4.

72. Yanny, B.; Gardner, S. The Stellar Number Density Distribution in the Local Solar Neighborhood is North-South Asymmetric. Astrophys. J. 2013, 777, doi:10.1088/0004-637X/777/2/91.

73. Steinmetz, M.; Zwitter, T.; Siebert, A.; Watson, F.G.; Freeman, K.C.; Munari, U.; Campbell, R.; Williams, M.; Seabroke, G.M.; Wyse, R.F.G.; et al. The Radial Velocity Experiment (RAVE): First Data Release. Astrophys. J. 2006, 132, 1645-1668.

74. Williams, M.E.K.; Steinmetz, M.; Binney, J.; Siebert, A.; Enke, H.; Famaey, B.; Minchev, I.; de Jong, R.S.; Boeche, C.; Freeman, K.C.; et al. The wobbly Galaxy: Kinematics north and south with RAVE red-clump giants. Mon. Not. R. Astron. Soc. 2013, 436, 101-121. 
75. Cui, X.Q.; Zhao, Y.H.; Chu, Y.Q.; Li, G.P.; Li, Q.; Zhang, L.P.; Su, H.J.; Yao, Z.Q.; Wang, Y.N.; Xing, X.Z.; et al. The Large Sky Area Multi-Object Fiber Spectroscopic Telescope (LAMOST). Res. Astron. Astrophys. 2012, 12, 1197-1242.

76. Deng, L.C.; Newberg, H.J.; Liu, C.; Carlin, J.L.; Beers, T.C.; Chen, L.; Chen, Y.Q.; Christlieb, N.; Grillmair, C.J.; Guhathakurta, P.; et al. LAMOST Experiment for Galactic Understanding and Exploration (LEGUE) - The survey's science plan. Res. Astron. Astrophys. 2012, 12, 735-754.

77. Zhao, G.; Zhao, Y.H.; Chu, Y.Q.; Jing, Y.P.; Deng, L.C. LAMOST spectral survey-An overview. Res. Astron. Astrophys. 2012, 12, 723-734.

78. Carlin, J.L.; DeLaunay, J.; Newberg, H.J.; Deng, L.; Gole, D.; Grabowski, K.; Jin, G.; Liu, C.; Liu, X.; Luo, A.L.; et al. Substructure in Bulk Velocities of Milky Way Disk Stars. Astrophys. J. Lett. 2013, 777, doi:10.1088/2041-8205/777/1/L5.

79. Widrow, L.M.; Barber, J.; Chequers, M.H.; Cheng, E. Bending and breathing modes of the Galactic disc. Mon. Not. R. Astron. Soc. 2014, 440, 1971-1981.

80. Weinberg, M.D.; Blitz, L. A Magellanic Origin for the Warp of the Galaxy. Astrophys. J. Lett. 2006, 641, L33-L36.

81. D'Onghia, E.; Madau, P.; Vera-Ciro, C.; Quillen, A.; Hernquist, L. Excitation of Coupled Stellar Motions in the Galactic Disk by Orbiting Satellites. Astrophys. J. 2016, 823, doi:10.3847/0004-637X/823/1/4.

82. Monari, G.; Famaey, B.; Siebert, A.; Grand, R.J.J.; Kawata, D.; Boily, C. The effects of bar-spiral coupling on stellar kinematics in the Galaxy. Mon. Not. R. Astron. Soc. 2016, 461, 3835-3846.

83. Gómez, F.A.; Minchev, I.; O'Shea, B.W.; Beers, T.C.; Bullock, J.S.; Purcell, C.W. Vertical density waves in the Milky Way disc induced by the Sagittarius dwarf galaxy. Mon. Not. R. Astron. Soc. 2013, 429, 159-164.

84. Li, J.; Newberg, H.J.; Carlin, J.L.; Deng, L.; Newby, M.; Willett, B.A.; Xu, Y.; Luo, Z. On Rings and Streams in the Galactic Anti-Center. Astrophys. J. 2012, 757, doi:10.1088/0004-637X/757/2/151.

85. Carlin, J.L.; Casetti-Dinescu, D.I.; Grillmair, C.J.; Majewski, S.R.; Girard, T.M. Kinematics in Kapteyn's Selected Area 76: Orbital Motions Within the Highly Substructured Anticenter Stream. Astrophys. J. 2010, 725, 2290-2311.

86. De Boer, T.J.L.; Belokurov, V.; Koposov, S.E. The fall of the Northern Unicorn: Tangential motions in the Galactic Anti-centre with SDSS and Gaia. Mon. Not. R. Astron. Soc. 2017, 1-16.

87. Gaia Collaboration.; Prusti, T.; de Bruijne, J.H.J.; Brown, A.G.A.; Vallenari, A.; Babusiaux, C.; Bailer-Jones, C.A.L.; Bastian, U.; Biermann, M.; Evans, D.W.; et al. The Gaia mission. Astron. Astrophys. 2016, 595, doi:10.1051/0004-6361/201629272.

88. Ivezic, Z.; Axelrod, T.; Brandt, W.N.; Burke, D.L.; Claver, C.F.; Connolly, A.; Cook, K.H.; Gee, P.; Gilmore, D.K.; Jacoby, S.H.; et al. Large Synoptic Survey Telescope: From Science Drivers To Reference Design. Serbian Astron. J. 2008, 176, 1-13.

89. Dalton, G.; Trager, S.; Abrams, D.C.; Bonifacio, P.; Aguerri, J.A.L.; Middleton, K.; Benn, C.; Dee, K.; Sayède, F.; Lewis, I.; et al. Final design and progress of WEAVE: The next generation wide-field spectroscopy facility for the William Herschel Telescope. In Proceedings of the SPIE Ground-based and Airborne Instrumentation for Astronomy VI, Edinburgh, UK, 26-30 June 2016; Volume 9908, p. $99081 \mathrm{G}$.

90. Walcher, C.J.; de Jong, R.S.; Dwelly, T.; Bellido, O.; Boller, T.; Chiappini, C.; Feltzing, S.; Irwin, M.; McMahon, R.; Merloni, A.; et al. 4MOST: Science operations for a large spectroscopic survey program with multiple science cases executed in parallel. In Proceedings of the SPIE Observatory Operations: Strategies, Processes, and Systems VI, Edinburgh, UK, 26 June-1 July 2016; Volume 9910, p. 99101N.

91. Tamura, N.; PFS Collaboration. Prime Focus Spectrograph (PFS): A Very Wide-Field, Massively Multi-Object, Optical and Near-Infrared Fiber-Fed Spectrograph on the Subaru Telescope. In Multi-Object Spectroscopy in the Next Decade: Big Questions, Large Surveys, and Wide Fields; Skillen, I., Barcells, M., Trager, S., Eds.; Astronomical Society of the Pacific Conference Series; Astronomical Society of the Pacific: San Francisco, CA, USA, 2016; Volume 507, p. 387.

92. Majewski, S.R.; APOGEE Team; APOGEE-2 Team. The Apache Point Observatory Galactic Evolution Experiment (APOGEE) and its successor, APOGEE-2. Astron. Nachr. 2016, 337, doi:10.1002/asna.201612387.

93. Majewski, S.R.; Ostheimer, J.C.; Rocha-Pinto, H.J.; Patterson, R.J.; Guhathakurta, P.; Reitzel, D. Detection of the Main-Sequence Turnoff of a Newly Discovered Milky Way Halo Structure in the Triangulum-Andromeda Region. Astrophys. J. 2004, 615, 738-743.

94. Ferguson, A.M.N.; Irwin, M.J.; Ibata, R.A.; Lewis, G.F.; Tanvir, N.R. Evidence for Stellar Substructure in the Halo and Outer Disk of M31. Astrophys. J. 2002, 124, 1452-1463. 
95. Ibata, R.; Chapman, S.; Ferguson, A.M.N.; Lewis, G.; Irwin, M.; Tanvir, N. On the Accretion Origin of a Vast Extended Stellar Disk around the Andromeda Galaxy. Astrophys. J. 2005, 634, 287-313.

96. Monachesi, A.; Bell, E.F.; Radburn-Smith, D.J.; Vlajić, M.; de Jong, R.S.; Bailin, J.; Dalcanton, J.J.; Holwerda, B.W.; Streich, D. Testing Galaxy Formation Models with the GHOSTS Survey: The Color Profile of M81's Stellar Halo. Astrophys. J. 2013, 766, doi:10.1088/0004-637X/766/2/106.

97. Crnojević, D.; Sand, D.J.; Spekkens, K.; Caldwell, N.; Guhathakurta, P.; McLeod, B.; Seth, A.; Simon, J.D.; Strader, J.; Toloba, E. The Extended Halo of Centaurus A: Uncovering Satellites, Streams, and Substructures. Astrophys. J. 2016, 823, doi:10.3847/0004-637X/823/1/19.

98. Ibata, R.; Martin, N.F.; Irwin, M.; Chapman, S.; Ferguson, A.M.N.; Lewis, G.F.; McConnachie, A.W. The Haunted Halos of Andromeda and Triangulum: A Panorama of Galaxy Formation in Action. Astrophys. J. 2007, 671, 1591-1623.

99. Harmsen, B.; Monachesi, A.; Bell, E.F.; de Jong, R.S.; Bailin, J.; Radburn-Smith, D.J.; Holwerda, B.W. Diverse stellar haloes in nearby Milky Way mass disc galaxies. Mon. Not. R. Astron. Soc. 2017, 466, 1491-1512.

100. Martínez-Delgado, D.; Gabany, R.J.; Crawford, K.; Zibetti, S.; Majewski, S.R.; Rix, H.W.; Fliri, J.; Carballo-Bello, J.A.; Bardalez-Gagliuffi, D.C.; Peñarrubia, J.; et al. Stellar Tidal Streams in Spiral Galaxies of the Local Volume: A Pilot Survey with Modest Aperture Telescopes. Astrophys. J. 2010, 140, 962-967.

101. Van Dokkum, P.G.; Abraham, R.; Merritt, A. First Results from the Dragonfly Telephoto Array: The Apparent Lack of a Stellar Halo in the Massive Spiral Galaxy M101. Astrophys. J. Lett. 2014, 782, doi:10.1088/2041-8205/782/2/L24.

102. Duc, P.A.; Cuillandre, J.C.; Karabal, E.; Cappellari, M.; Alatalo, K.; Blitz, L.; Bournaud, F.; Bureau, M.; Crocker, A.F.; Davies, R.L.; et al. The ATLAS ${ }^{3 D}$ project - XXIX. The new look of early-type galaxies and surrounding fields disclosed by extremely deep optical images. Mon. Not. R. Astron. Soc. 2015, 446, 120-143.

103. Spavone, M.; Capaccioli, M.; Napolitano, N.R.; Iodice, E.; Grado, A.; Limatola, L.; Cooper, A.; Cantiello, M.; Forbes, D.A.; Paolillo, M.; et al. VEGAS: A VST Early-type GAlaxy Survey. II. Photometric study of giant ellipticals and their stellar halos. Astron. Astrophys. 2017, doi:10.1051/0004-6361/201629111.

104. Spergel, D.; Gehrels, N.; Breckinridge, J.; Donahue, M.; Dressler, A.; Gaudi, B.S.; Greene, T.; Guyon, O.; Hirata, C.; Kalirai, J.; et al. WFIRST-2.4: What Every Astronomer Should Know. ArXiv 2013, arXiv:1305.5425.

105. Toth, G.; Ostriker, J.P. Galactic disks, infall, and the global value of Omega. Astrophys. J. 1992, 389, 5-26.

106. Quinn, P.J.; Hernquist, L.; Fullagar, D.P. Heating of galactic disks by mergers. Astrophys. J. 1993, 403, 74-93.

107. Walker, I.R.; Mihos, J.C.; Hernquist, L. Quantifying the Fragility of Galactic Disks in Minor Mergers. Astrophys. J. 1996, 460, doi:10.1086/176956.

108. Velazquez, H.; White, S.D.M. Sinking satellites and the heating of galaxy discs. Mon. Not. R. Astron. Soc. 1999, 304, 254-270.

109. Font, A.S.; Navarro, J.F.; Stadel, J.; Quinn, T. Halo Substructure and Disk Heating in a $\Lambda$ Cold Dark Matter Universe. Astrophys. J. Lett. 2001, 563, L1-L4.

110. Ardi, E.; Tsuchiya, T.; Burkert, A. Constraints of the Clumpiness of Dark Matter Halos through Heating of the Disk Galaxies. Astrophys. J. 2003, 596, 204-215.

111. Benson, A.J.; Lacey, C.G.; Frenk, C.S.; Baugh, C.M.; Cole, S. Heating of galactic discs by infalling satellites. Mon. Not. R. Astron. Soc. 2004, 351, 1215-1236.

112. Stewart, K.R.; Bullock, J.S.; Wechsler, R.H.; Maller, A.H.; Zentner, A.R. Merger Histories of Galaxy Halos and Implications for Disk Survival. Astrophys. J. 2008, 683, 597-610.

113. Hopkins, P.F.; Hernquist, L.; Cox, T.J.; Younger, J.D.; Besla, G. The Radical Consequences of Realistic Satellite Orbits for the Heating and Implied Merger Histories of Galactic Disks. Astrophys. J. 2008, 688, 757-769.

114. Villalobos, Á.; Helmi, A. Simulations of minor mergers-I. General properties of thick discs. Mon. Not. R. Astron. Soc. 2008, 391, 1806-1827.

115. Purcell, C.W.; Kazantzidis, S.; Bullock, J.S. The Destruction of Thin Stellar Disks Via Cosmologically Common Satellite Accretion Events. Astrophys. J. Lett. 2009, 694, L98-L102.

116. Kazantzidis, S.; Zentner, A.R.; Kravtsov, A.V.; Bullock, J.S.; Debattista, V.P. Cold Dark Matter Substructure and Galactic Disks. II. Dynamical Effects of Hierarchical Satellite Accretion. Astrophys. J. 2009, 700, 1896-1920.

117. Sachdeva, S.; Saha, K. Survival of Pure Disk Galaxies over the Last 8 Billion Years. Astrophys. J. Lett. 2016, 820, doi:10.3847/2041-8205/820/1/L4.

118. Moetazedian, R.; Just, A. Impact of cosmological satellites on the vertical heating of the Milky Way disc. Mon. Not. R. Astron. Soc. 2016, 459, 2905-2924. 
119. Abadi, M.G.; Navarro, J.F.; Steinmetz, M. Stars beyond galaxies: The origin of extended luminous haloes around galaxies. Mon. Not. R. Astron. Soc. 2006, 365, 747-758.

120. Zolotov, A.; Willman, B.; Brooks, A.M.; Governato, F.; Brook, C.B.; Hogg, D.W.; Quinn, T.; Stinson, G. The Dual Origin of Stellar Halos. Astrophys. J. 2009, 702, 1058-1067.

121. Zolotov, A.; Willman, B.; Brooks, A.M.; Governato, F.; Hogg, D.W.; Shen, S.; Wadsley, J. The Dual Origin of Stellar Halos. II. Chemical Abundances as Tracers of Formation History. Astrophys. J. 2010, 721, 738-743.

122. Font, A.S.; McCarthy, I.G.; Crain, R.A.; Theuns, T.; Schaye, J.; Wiersma, R.P.C.; Dalla Vecchia, C. Cosmological simulations of the formation of the stellar haloes around disc galaxies. Mon. Not. R. Astron. Soc. 2011, 416, 2802-2820.

123. McCarthy, I.G.; Font, A.S.; Crain, R.A.; Deason, A.J.; Schaye, J.; Theuns, T. Global structure and kinematics of stellar haloes in cosmological hydrodynamic simulations. Mon. Not. R. Astron. Soc. 2012, 420, 2245-2262.

124. Tissera, P.B.; Scannapieco, C.; Beers, T.C.; Carollo, D. Stellar haloes of simulated Milky-Way-like galaxies: Chemical and kinematic properties. Mon. Not. R. Astron. Soc. 2013, 432, 3391-3400.

125. Tissera, P.B.; Beers, T.C.; Carollo, D.; Scannapieco, C. Stellar haloes in Milky Way mass galaxies: From the inner to the outer haloes. Mon. Not. R. Astron. Soc. 2014, 439, 3128-3138.

126. Pillepich, A.; Madau, P.; Mayer, L. Building Late-type Spiral Galaxies by In-situ and Ex-situ Star Formation. Astrophys. J. 2015, 799, doi:10.1088/0004-637X/799/2/184.

127. Cooper, A.P.; Parry, O.H.; Lowing, B.; Cole, S.; Frenk, C. Formation of in situ stellar haloes in Milky Way-mass galaxies. Mon. Not. R. Astron. Soc. 2015, 454, 3185-3199.

128. Carollo, D.; Beers, T.C.; Lee, Y.S.; Chiba, M.; Norris, J.E.; Wilhelm, R.; Sivarani, T.; Marsteller, B.; Munn, J.A.; Bailer-Jones, C.A.L.; et al. Two stellar components in the halo of the Milky Way. Nature 2007, 450, 1020-1025.

129. Deason, A.J.; Belokurov, V.; Evans, N.W.; Johnston, K.V. Broken and Unbroken: The Milky Way and M31 Stellar Halos. Astrophys. J. 2013, 763, 113.

130. Dorman, C.E.; Widrow, L.M.; Guhathakurta, P.; Seth, A.C.; Foreman-Mackey, D.; Bell, E.F.; Dalcanton, J.J.; Gilbert, K.M.; Skillman, E.D.; Williams, B.F. A New Approach to Detailed Structural Decomposition from the SPLASH and PHAT Surveys: Kicked-up Disk Stars in the Andromeda Galaxy? Astrophys. J. 2013, 779, doi:10.1088/0004-637X/779/2/103.

131. Hawkins, K.; Kordopatis, G.; Gilmore, G.; Masseron, T.; Wyse, R.F.G.; Ruchti, G.; Bienaymé, O.; Bland-Hawthorn, J.; Boeche, C.; Freeman, K.; et al. Characterizing the high-velocity stars of RAVE: The discovery of a metal-rich halo star born in the Galactic disc. Mon. Not. R. Astron. Soc. 2015, 447, 2046-2058.

132. Bonaca, A.; Conroy, C.; Wetzel, A.; Hopkins, P.F.; Keres, D. Gaia reveals a metal-rich in-situ component of the local stellar halo. Astrophys. J. 2017, doi:10.3847/1538-4357/aa7d0c.

133. Chakrabarti, S.; Blitz, L. Tidal imprints of a dark subhalo on the outskirts of the Milky Way. Mon. Not. R. Astron. Soc. 2009, 399, L118-L122.

134. Chakrabarti, S.; Bigiel, F.; Chang, P.; Blitz, L. Finding Dwarf Galaxies from Their Tidal Imprints. Astrophys. J. 2011, 743, doi:10.1088/0004-637X/743/1/35.

135. Chang, P.; Chakrabarti, S. Dark subhaloes and disturbances in extended H I discs. Mon. Not. R. Astron. Soc. 2011, 416, 618-628.

(C) 2017 by the authors. Licensee MDPI, Basel, Switzerland. This article is an open access article distributed under the terms and conditions of the Creative Commons Attribution (CC BY) license (http:// creativecommons.org/licenses/by/4.0/). 